\title{
Retrospective analysis of the outcomes of endoscopic submucosal dissection for the diagnosis and treatment of subepithelial lesions in a center with high expertise
}

\author{
João Santos-Antunesa,b, Margarida Marquesa , Rui Morais ${ }^{a}$, Francisco Baldaque-Silvac, Filipe Vilas-Boas ${ }^{a}$, \\ Pedro Moutinho-Ribeiro a, Susana Lopes ${ }^{\mathrm{a}}$, Fátima Carneirod, Guilherme Macedo ${ }^{\mathrm{a}}$
}

Centro Hospitalar S. João, Porto, Portugal; Instituto de Investigação e Inovação em Saúde da Universidade do Porto; Karolinska University Hospital and Karolinska Institute, Stockholm, Sweden

Abstract

${ }^{a}$ Gastroenterology Department, Faculty of Medicine, Centro Hospitalar S. João, Porto, Portugal (João Santos-Antunes, Margarida Marques, Rui Morais, Filipe Vilas-Boas ${ }^{\mathrm{a}}$, Pedro Moutinho-Ribeiro, Susana Lopes, Guilherme Macedo); ' Ipatimup/i3S (Instituto de Investigação e Inovação em Saúde da Universidade do Porto), Porto, Portugal (João Santos-Antunes); ${ }^{\mathrm{C}}$ Division of Medicine, Department of Upper Gastrointestinal Diseases, Karolinska University Hospital and Karolinska Institute, Stockholm, Sweden (Francisco Baldaque-Silva); dPathology Department, Faculty of Medicine, Centro Hospitalar S. João, Porto, Portugal (Fátima Carneiro)

Conflict of Interest: None

Correspondence to: João Santos-Antunes, Gastroenterology Department, Centro Hospitalar S. João, Al. Prof. Hernani Monteiro, 4200-319, Porto, Portugal, e-mail: joao.claudio.antunes@gmail.com

Received 14 July 2021; accepted 26 August 2021; published online 10 November 2021

DOI: https://doi.org/10.20524/aog.2021.0675

\section{Introduction}

Subepithelial lesions (SELs) are commonly found in routine endoscopic procedures. They are usually an incidental finding, since most of them are asymptomatic. Data regarding their epidemiology, natural history, and optimal diagnosis or management are still insufficient. Clinical decisions are made considering the malignant potential, difficult to predict, and the certainty of the endoscopic ultrasound or imaging diagnosis, since histological characterization is still difficult to obtain in a considerable proportion of these lesions [1]. SELs can be resected by endoscopic techniques or by surgery. Endoscopic treatment, namely endoscopic submucosal dissection (ESD), is less invasive than surgery, and can be applied for both diagnostic and therapeutic interventions in SELs [2-4]. 
ESD is indeed a well-established technique for the treatment of early epithelial neoplasia in the gastrointestinal tract $[5,6]$. Despite its efficacy in epithelial lesions, its efficacy in SELs has only been evaluated in smaller series and is even somewhat controversial in some cases $[7,8]$. SELs can be resected using the classical ESD technique, or by applying some variants, such as submucosal tunneling endoscopic resection (STER) [9]. Initially, only superficial lesions (mucosal or submucosal) were selected, but many reports have been described for lesions located in the muscularis propria [10], using ESD, STER, or full-thickness resection [9]. Techniques for the closure of the deliberate perforation range from simple clipping to endoscopic suturing [11]. Furthermore, laparoscopic-endoscopic procedures, preferably without communication between the lumen and extraluminal cavities, have been described $[12,13]$.

Data from endoscopic treatment for SELs in the West is still scarce. Therefore, results from large real-life series are very useful. In this study, we aimed to assess the diagnostic and therapeutic utility of ESD for the management of SELs in a tertiary ESD referral center.

\section{Patients and methods}

\section{Patient selection, lesion evaluation and ESD technique}

ESDs included in this study were performed in our center from November 2010 to February 2021. Patients' data were entered into an electronic database, and information about ESD, pathological result and follow up was prospectively recorded. Some of the lesions included in this study were briefly enumerated in our previous series [14]. Written informed consent was obtained from every patient before ESD.

ESDs were performed by 3 endoscopists (JSA, MM, FBS). For the purposes of this study, we selected only those ESDs performed on SELs. Lesions were initially evaluated using highdefinition endoscopes (GIF-Q180, -H180 or -H190 gastroscopes, with Exera-II or Exera-III processors, Olympus ${ }^{\circledR}$, Tokyo, Japan) and, whenever considered necessary, endoscopic ultrasound (EUS) evaluation was performed. For lesions $<1 \mathrm{~cm}$, the radial echoendoscope (Pentax ${ }^{\circledR}$ EG-3670URK) was used for tumor characterization. Whenever the need for EUS-tissue acquisition was anticipated (lesions $>15-20 \mathrm{~mm}$ ), the linear echoendoscope was selected (Olympus ${ }^{\circledR}$ GF-UCT180, Olympus ${ }^{\circledR}$ GF-UC140 or Pentax ${ }^{\circledR}$ EG3870UTK). Echoendoscopes were coupled with HITACHI HI VISION Avius and Olympus EU-ME2 ultrasound processors. EUS with fine needle aspiration (FNA) was performed using 22- or 25-G needles, according to lesion location and size.

Lesions were selected for ESD if located in the muscularis mucosa or submucosa. Dissection was performed using 1.5- or 2-mm Dual-knives (Olympus ${ }^{\circledR}$, Tokyo, Japan) for mucosal incision. DualKnives, insulated tip (IT)-2 or IT-nano knives (Olympus $^{\circledR}$, Tokyo, Japan) were used for submucosal dissection. Erbe ICC-200, ICC-300 or VIO-300 electrosurgical units (ERBE ${ }^{\circledR}$ Elektromedizin GmBH, Tubingen, Germany) were used, with Endocut Mode effect 2 for mucosal incision and Forced or Swift coagulation (effect 3, $30 \mathrm{~W}$ ) for submucosal dissection.
Coagrasper (Olympus ${ }^{\circledR}$, Tokyo, Japan) was used for hemostasis (effect 5, $80 \mathrm{~W}$ ) whenever necessary. Hybrid ESD was performed at the endoscopist's discretion, using a circumferential incision with a DualKnife and further resection with a snare. Traction was used whenever necessary, by applying a clip with a wire (clip-and-wire technique) or with a snare (yo-yo technique [15]) in the border of the partially dissected lesion, allowing traction by pulling on the wire or the snare externally to the patient.

\section{Histopathological evaluation}

ESD specimens were sent for pathology evaluation with pins on a cork plate, fixed in formalin. Sectioning at 2-mm intervals were performed to evaluate lateral and vertical margins. All the specimens were analyzed by 2 expert gastrointestinal pathologists.

\section{Definitions and outcomes}

ESD was considered a failure whenever the target lesion was not removed. En-bloc resection was defined as retrieval of the target lesion in a single specimen, and piecemeal resection if the lesion was removed in more than one fragment. R0 resection was recorded when pathological evaluation showed free horizontal and vertical margins in an en-bloc resected specimen. Resection of an SEL was considered curative if it was removed with free margins, without lymphovascular invasion. Procedure-related mortality was defined as any death consequent to the ESD procedure.

\section{Statistical analysis}

Categorical variables were described as absolute (n) and relative frequencies (\%). Mean and standard deviation, or median and percentiles or range, were used for continuous variables, as appropriate. When testing a hypothesis involving continuous variables, the Student's $t$-test or Mann-Whitney were used as appropriate, considering normality assumptions and the number of groups compared. When testing a hypothesis about categorical variables a chi-square test and Fisher's exact test were used, as appropriate. The significance level used was 0.05 . Statistical analysis was performed using the Statistical Package for the Social Sciences v.25.

\section{Results}

\section{General description}

Six hundred thirty-four ESDs were performed and evaluated retrospectively. Of these, 55 (9\%) were performed in SELs, in 24 male patients (44\%), with a mean age of $56 \pm 15$ years; their general description is presented in Table 1. Lesions were located in the esophagus $(n=6)$, stomach $(n=34)$, and rectum 
$(\mathrm{n}=15)$. Median lesion size was $22 \mathrm{~mm}$ (interquartile range [IQR] 25-75\% 14-31 mm). Median procedure time was $60 \mathrm{~min}$ (IQR 25-75\% 40-120 min).

Hybrid ESD was performed in 4 cases, and traction using clip-and-wire or yo-yo techniques was used in 3 cases.

The target lesion was not removed in 2 procedures (ESD failures, 4\%): 1 granular cell tumor in the esophagus and 1 neuroendocrine tumor (NET) in the stomach, because of perforation (closure with endoscopic clipping) and adherence to the muscle layer (interrupted procedure), respectively.

Most procedures (59\%) were performed in an ambulatory setting, with patients discharged on the same day. Most of the admitted patients were hospitalized for $24 \mathrm{~h} \mathrm{(70 \% ),} \mathrm{and} \mathrm{the}$ longest admission time was 10 days. Two patients developed low-grade fever, one patient had minor bleeding detected during same-day second-look endoscopy, and one patient had bleeding after discharge needing readmission.

Surgery or blood transfusion consequent to an ESD adverse event was not needed in any case, and procedure-related mortality was $0 \%$.

\section{Diagnostic workup}

SELs were detected in the vast majority of the cases by routine upper endoscopy or colonoscopy in asymptomatic patients, since only 2 patients had symptoms, namely dysphagia (a giant esophageal lipoma [16]) and vomiting (a gastric lipoma resulting in "ball-valve syndrome").

Of the 55 ESDs for SEL resection, 45 (82\%) had a previous EUS evaluation. Reasons not to perform EUS were mainly related to the small size of some of the lesions, namely small gastric NETs ( $n=6$ ), to the good lifting of some polypoid lesions, directly removed by ESD ( $n=3)$, or to the very superficial laterally spreading tumor-like lesions (rectal Schwannoma). Four

Table 1 Characteristics of endoscopic submucosal dissection procedures $(\mathrm{n}=55)$

\begin{tabular}{lc}
\hline Characteristics & Value \\
\hline Lesion size (mm), median (IQR 25-75\%) & $22(14-31)$ \\
Overall & $15(14-84)$ \\
Esophagus & $25(20-33)$ \\
Stomach & $17(15-22)$ \\
Rectum & \\
Procedure time (min), median (IQR 25-75\%) & $60(40-120)$ \\
Overall & $105(63-409)$ \\
Esophagus & $60(46-120)$ \\
Stomach & $43(19-68)$ \\
Rectum & \\
R0 resection rate $(\%)^{*}$ & 65 \\
Overall & 100 \\
Esophagus $(\mathrm{n}=2)$ & 50 \\
Stomach $(\mathrm{n}=10)$ & 75 \\
Rectum $(\mathrm{n}=8)$ & \\
\hline
\end{tabular}

For neuroendocrine tumors, gastrointestinal stromal tumors and granular cell tumors

$I Q R$, interquartile range patients had complementary staging by computed tomography scan previous to the ESD.

Only 11 patients (20\%) had a pathological diagnosis before ESD. The diagnosis was obtained by "bite-on-bite" forceps biopsies in the majority of the cases $(n=10)$; this technique was in fact diagnostic in most of the cases where it was attempted $(10 / 14,71 \%)$. Of the 45 patients who underwent EUS, only 2 (4\%) had FNA; of those, 1 was diagnostic (gastric NET) and 1 was inconclusive.

\section{ESD for the treatment of SELs with malignant potential}

Of the 53 successful ESDs on SELs, 14 (26\%) were performed on NETs, $3(6 \%)$ on granular cell tumors, and $3(6 \%)$ on gastrointestinal stromal tumors (GIST) (Table 2). These 20 lesions were removed en bloc in 100\% of the cases, including 13 (65\%) R0 resections/curative resections. Reasons for non-curative resection were positive deep margin in 4 cases ( 1 GIST, 2 NETs, 1 granular cell tumor) and positive deep and lateral margins in 3 cases (NETs). The decision whether to operate on patients with noncurative ESD was taken in a multidisciplinary team conference, after which 3 patients (NETs) underwent surgery, with 2 subtotal gastrectomies and 1 abdominoperineal amputation; one of them (gastric NET) had 2 positive lymph nodes, and none of them had a lesion on the wall of the surgical specimen. Regarding the other 4 patients, only one had a long-term endoscopic follow up (64 weeks), and 2 had short-term follow up (5 and 24 weeks), none of them with recurrence.

One patient (greater curvature of gastric body) needed hemostasis with clips during ESD. No delayed bleeding or perforation was recorded in this group.

Table 2 Pathological results of specimens from 53 successful endoscopic submucosal dissection procedures

\begin{tabular}{lc}
\hline Pathological results & Number \\
\hline Esophagus (n) & 5 \\
Granular cell tumor & 2 \\
Leiomyoma & 1 \\
Lipoma & 1 \\
Schwannoma & 1 \\
Stomach (n) & 33 \\
Inflammatory fibroid polyp & 10 \\
Neuroendocrine tumors & 6 \\
Ectopic pancreas & 6 \\
Lipoma & 4 \\
Gastrointestinal stromal tumors & 3 \\
Enterochromaffin cell & 2 \\
hyperplasia & \\
Granular cell tumor & 1 \\
Gastric antral vascular ectasia & 1 \\
Rectum (n) & 15 \\
Neuroendocrine tumors & 8 \\
Leiomyoma & 2 \\
Lipoma & 2 \\
Cyst (rectitis profunda) & 1 \\
Schwannoma & 1 \\
Pseudotumoral calcified lesion & 1 \\
\hline
\end{tabular}




\section{ESD for the diagnosis and treatment of other SELs}

Thirty-three ESDs were performed in non-neoplastic SELs or in neoplastic SELS with none or very low malignant potential, in lesions in which previous diagnostic workup was inconclusive (Table 2). The reasons for ESD in these lesions were the uncertainty of the diagnosis after EUS evaluation and, in 1 case, also the lesion growth between 2 EUS (an ectopic pancreas). Of the lipomas, 2 were symptomatic and the remaining had atypical findings, being described as hypo or isoechoic lesions, some of them heterogeneous (Fig. 1). Regarding inflammatory fibroid polyps and ectopic pancreas, the differential diagnosis with NETs or GIST could not be clarified after EUS evaluation, so ESD was decided upon. One calcified rectal lesion was very similar to a rectal NET lesion [17] and the rectal Schwannoma presented as a laterally spreading tumor-like lesion [18].

There were 2 delayed bleedings (1 gastric inflammatory fibroid polyp and 1 gastric lipoma), both managed by endoscopy with clips. There were no cases of perforation.

\section{Discussion}

This represents one of the largest western ESD series regarding the management of SELs by ESD. Eligibility criteria for SEL resection based on high-quality evidence do not exist [19]. However, there are groups of patients who are usually selected for treatment: symptomatic SELs, GISTs with more than $2 \mathrm{~cm}$ or with high-risk features, NETs, other SELs with rapid growth or with worrisome ultrasonographic or histological features, and undetermined lesions, mainly in younger patients $[8,20]$.

In our series, only 2 patients had a symptomatic SEL (2 lipomas); in fact, it was the group of patients with

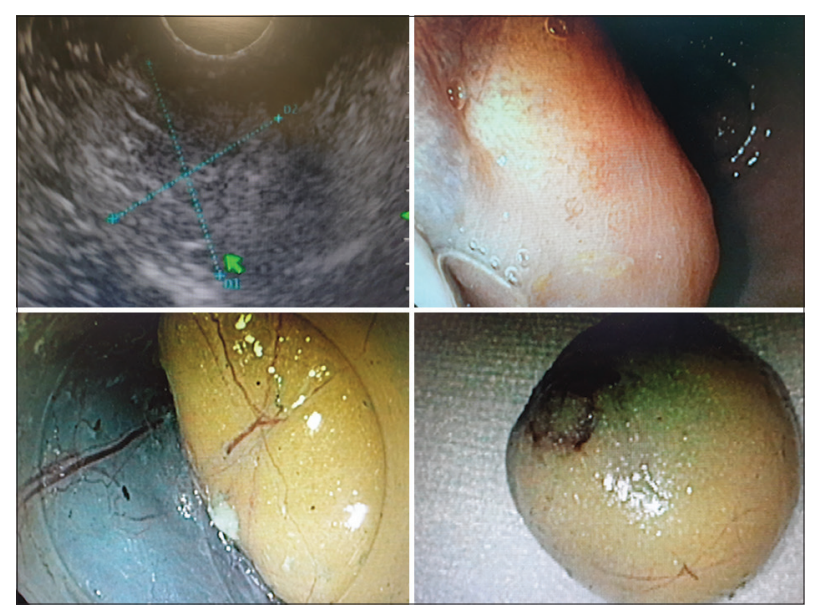

Figure 1 Lesion on the distal rectum, described on endoscopic ultrasound as an isoechogenic, heterogeneous lesion in the submucosa, compatible with a neuroendocrine tumor. During endoscopic submucosal dissection, a soft, yellowish lesion was observed, typical of a lipoma, confirmed in the pathological evaluation undetermined diagnosis after EUS that comprised a significant portion of our cases in the real-life scenario. The optimal management in this group is uncertain and several aspects must be weighed, namely the patient's age (crucial for the decision because of the long-term risk of progression, impossible to predict in undetermined lesions, and the need for multiple evaluation procedures, with upper endoscopies and EUS, to address morphological changes and dimensional progression), the presence of atypical features, and the ease and feasibility of performing minimally invasive endoscopic resection techniques or, on the other hand, whether large surgical procedures are unavoidable given the SEL size or location. In our series these lesions comprised $60 \%$ of the ESDs. This can be explained by the large experience of our center in ESD, which has lowered our threshold for offering endoscopic resection for either diagnostic or therapeutic purposes. In addition, this allows patients to be discharged from the outpatient clinic, and avoids the repetition of multiple diagnostic procedures, as well as contributing to alleviating patients' anxiety through the resolution of a previously undiagnosed lesion. Furthermore, ESD was demonstrated to be very safe in this group of patients.

Most of our patients had an EUS evaluation previous to ESD. This is very useful in the planning of ESD, as it assesses the layer of origin, and for attempts at characterization and diagnosis [3]. We offered ESD to those patients whose diagnosis could not be determined with certainty by the endoscopist, but included neoplastic lesions, which have an indication for removal, in its differential diagnosis. For instance, none of the asymptomatic lipomas removed by ESD had the typical hyperechogenic, homogeneous, well-delimited lesions in the third layer; they were described as hypo- or isoechogenic, heterogeneous lesions. FNA was only attempted in 2 cases; this is explained by the typical lower accuracy in SELs compared to extraluminal lesions and the possibility of ESD in our center, which leads endoscopists to perform fewer FNA attempts.

Pre-ESD diagnosis is an important issue to discuss and it could be very useful, since it can help the endoscopist better plan the therapeutic strategy and technique. In our study, pathological diagnosis was achieved in the majority of cases in which "bite-on-bite" biopsies were attempted. This apparently high acuity of the technique is probably due to the fact that it was preferably performed in lesions that had a very superficial appearance endoscopically, namely in esophageal granular cell tumors or gastric NETs. Regarding pre-evaluation by EUS, the prediction of the layer of origin was described in a large study to be less than $75 \%$, which can compromise the success of the ESD, and diagnosis accuracy was around 83\% [21]. Furthermore, the selection of the lesions for ESD is not straightforward, and even scientific societies do not fully agree on the definition of high-risk EUS criteria [22].

Despite these limitations on pre-ESD evaluation, ESD has been showing promising results for the management of SELs [22-24]. In the literature we found very acceptable results for GISTs [25], NETs [8], and undetermined lesions [26]. Our rate of curative resections for this kind of lesions was lower than in ESDs performed for epithelial lesions [14]. This is due to the 
very shallow margin that we are able to maintain below the SELs and the difficulty for the pathologist to address the integrity of the entire margin around the lesion, adding to those cases in which we verify adherence to muscularis propria, which raises the risk of incomplete resection. Our rates of curative resection are in accordance to those previously reported in other series [8]. Interestingly, none of the patients with incomplete resection had residual lesions on the wall in the follow up, though one of them demonstrated metastatic lymph nodes.

Our study had some limitations. It was a retrospective evaluation, and included ESD procedures performed from the beginning of ESD practice in our department, and by 3 endoscopists; this represents our real-life clinical practice, but leads to a higher heterogeneity in terms of ESD technique and outcomes. Moreover, most of the lesions have not yet had a long-term follow up, in some cases because patients were lost to follow up; although it was not one of the main aims of the study, we cannot discard the possibility of long-term recurrence in patients who underwent endoscopic treatment, especially those without a curative resection. Finally, our results came from a high-volume center and should not be extrapolated to other centers with less experience in ESD. However, we think that our results show that endoscopists in those lower-volume centers can progress to ESD in SELs safely in the course of their learning curve.

In conclusion, ESD can be an effective treatment for SELs, even with lower curative rates comparing to procedures on epithelial lesions. Furthermore, it could be a safe and extremely valuable tool for the diagnosis and management of SELs in which diagnosis remains obscure after EUS.

\section{Summary Box}

\section{What is already known:}

- Subepithelial lesions (SELs) are commonly found in routine endoscopic procedures

- Data regarding natural history or optimal diagnosis or management are still insufficient

- Endoscopic therapy can be applied for resection of SELs

\section{What the new findings are:}

- In our clinical practice, only $20 \%$ had a pathological diagnosis before endoscopic submucosal dissection (ESD)

- None of the patients who underwent surgery because of non-curative ESD had lesions on the gastrointestinal wall

- The majority (62\%) of the ESDs on SELs were performed for the definite diagnosis of benign lesions or lesions with low malignant potential

\section{References}

1. Kim GH, Cho YK, Kim EY, et al; Korean EUS Study Group. Comparison of 22-gauge aspiration needle with 22-gauge biopsy needle in endoscopic ultrasonography-guided subepithelial tumor sampling. Scand J Gastroenterol 2014;49:347-354.

2. Bhagat VH, Kim M, Kahaleh M. A review of endoscopic fullthickness resection, submucosal tunneling endoscopic resection, and endoscopic submucosal dissection for resection of subepithelial lesions. J Clin Gastroenterol 2021;55:309-315.

3. McCarty TR, Ryou M. Endoscopic diagnosis and management of gastric subepithelial lesions. Curr Opin Gastroenterol 2020;36:530-537.

4. Dhaliwal A, Kolli S, Dhindsa BS, et al. Diagnostic yield of deep biopsy via endoscopic submucosal dissection for the diagnosis of upper gastrointestinal subepithelial tumors: a systematic review and meta-analysis. Ann Gastroenterol 2020;33:30-37.

5. Fujiya M, Tanaka K, Dokoshi T, et al. Efficacy and adverse events of EMR and endoscopic submucosal dissection for the treatment of colon neoplasms: a meta-analysis of studies comparing EMR and endoscopic submucosal dissection. Gastrointest Endosc 2015;81:583-595.

6. Park YM, Cho E, Kang HY, et al. The effectiveness and safety of endoscopic submucosal dissection compared with endoscopic mucosal resection for early gastric cancer: a systematic review and metaanalysis. Surg Endosc 2011;25:2666-2677.

7. Marcella C, Sarwar S, Ye H, Shi RH. Efficacy and safety of endoscopic treatment for gastrointestinal stromal tumors in the upper gastrointestinal tract. Clin Endosc 2020;53:458-465.

8. Kobara H, Miyaoka Y, Ikeda Y, et al. Outcomes of endoscopic submucosal dissection for subepithelial lesions localized within the submucosa, including neuroendocrine tumors: a multicenter prospective study. J Gastrointestin Liver Dis 2020;29:41-49.

9. Aslanian HR, Sethi A, Bhutani MS, et al; ASGE Technology Committee. ASGE guideline for endoscopic full-thickness resection and submucosal tunnel endoscopic resection. VideoGIE 2019;4:343-350.

10. Zhang Y, Peng JB, Mao XL, et al. Endoscopic resection of large $(\geq 4 \mathrm{~cm}$ ) upper gastrointestinal subepithelial tumors originating from the muscularis propria layer: a single-center study of 101 cases (with video). Surg Endosc 2021;35:1442-1452.

11. Kantsevoy SV, Bitner M, Mitrakov AA, Thuluvath PJ. Endoscopic suturing closure of large mucosal defects after endoscopic submucosal dissection is technically feasible, fast, and eliminates the need for hospitalization (with videos). Gastrointest Endosc 2014;79:503-507.

12. Goto O, Takeuchi H, Sasaki M, et al. Laparoscopy-assisted endoscopic full-thickness resection of gastric subepithelial tumors using a nonexposure technique. Endoscopy 2016;48:1010-1015.

13. Chai N, Du C, Gao Y, et al. Comparison between submucosal tunneling endoscopic resection and video-assisted thoracoscopic enucleation for esophageal submucosal tumors originating from the muscularis propria layer: a randomized controlled trial. Surg Endosc 2018;32:3364-3372.

14. Santos-Antunes J, Baldaque-Silva F, Marques M, Lopes J, Carneiro F, Macedo G. Real-life evaluation of the safety, efficacy and therapeutic outcomes of endoscopic submucosal dissection in a Western tertiary centre. United European Gastroenterol J 2018;6:702-709.

15. Baldaque-Silva F, Vilas-Boas F, Velosa M, Macedo G. Endoscopic submucosal dissection of gastric lesions using the "yo-yo technique". Endoscopy 2013;45:218-221.

16. Baldaque-Silva F, Marques M, Sanchez-Hernandez E, et al. Endoscopic submucosal dissection of a giant esophageal lipoma. Am J Gastroenterol 2016;111:1680.

17. Santos-Antunes J, Marques M, Andrade P, et al. Rectal calcified 
fibrous tumor diagnosed after endoscopic submucosal dissection of a suspected neuroendocrine tumor. Am J Gastroenterol 2016;111:764.

18. Gaspar R, Santos-Antunes J, Marques M, et al. Endoscopic submucosal dissection of a schwann cell hamartoma mimicking a lateral spreading tumor of the rectum. Acta Gastroenterol Belg 2017;80:429.

19. Longcroft-Wheaton G, Bhandari P. Endoscopic resection of submucosal tumors. Expert Rev Gastroenterol Hepatol 2015;9:659-669

20. Zhang X, Modayil R, Criscitelli T, Stavropoulos SN. Endoscopic resection for subepithelial lesions-pure endoscopic full-thickness resection and submucosal tunneling endoscopic resection. Transl Gastroenterol Hepatol 2019;4:39.

21. Li DM, Ren LL, Jiang YP. Long-term outcomes of endoscopic resection for gastric subepithelial tumors. Surg Laparosc Endosc Percutan Tech 2020;30:187-191.

22. Cho JW; Korean ESD Study Group. Current guidelines in the management of upper gastrointestinal subepithelial tumors. Clin Endosc 2016;49:235-240.

23. Godat S, Robert M, Caillol F, et al. Efficiency and safety of endoscopic resection in the management of subepithelial lesions of the stomach. United European Gastroenterol J 2016;4:250-256.

24. Bang CS, Baik GH, Shin IS, Suk KT, Yoon JH, Kim DJ. Endoscopic submucosal dissection of gastric subepithelial tumors: a systematic review and meta-analysis. Korean J Intern Med 2016;31:860-871.

25. He G, Wang J, Chen B, et al. Feasibility of endoscopic submucosal dissection for upper gastrointestinal submucosal tumors treatment and value of endoscopic ultrasonography in pre-operation assess and post-operation follow-up: a prospective study of 224 cases in a single medical center. Surg Endosc 2016;30:4206-4213.

26. Tae HJ, Lee HL, Lee KN, et al. Deep biopsy via endoscopic submucosal dissection in upper gastrointestinal subepithelial tumors: a prospective study. Endoscopy 2014;46:845-850. 